\title{
VIRTUAL COMMUNITY SUPPORT IN TELECARE
}

\author{
Hamideh Afsarmanesh, Víctor Guevara Masís, L.O. Hertzberger \\ University of Amsterdam \\ Kruislaan 403, 1098 SJ Amsterdam, THE NETHERLANDS \\ (hamideh,vguevara,bob)@science.uva.nl
}

\begin{abstract}
A few virtual community support systems, with some unsophisticated tools, appeared at the beginning of the era of Internet availability and pervasive computing. Ever since however, many such systems have emerged focusing on classified interests and motivations, and aiming to give people more possibilities to socialize and keep in touch with each other. A growing population in Europe, the elderly, can benefit from the provision of such virtual communities and their services in order to maintain and promote their independent living, and to support their optimal mental, emotional, and physical health and well being. The development of virtual community service in TeleCARE is based on multi-agent systems, and in particular the mobile agents provide a rich paradigm for information brokerage in networked environments. This paper provides an overview of Virtual Communities and its benefits, it also briefly describes the design of a support service on top of a multi agent infrastructure, developed within the TeleCARE project', to facilitate elderly's participation in virtual communities.
\end{abstract}

\section{INTRODUCTION}

Due to the lack of a formal definition, virtual communities are casually defined as distributed online services connecting a group of people that gather to keep in touch, focused on some common interest or purpose. As such, a virtual community can play an important role in the lives of its members, and thus may require proper management by those who own and maintains its services.

Within a focussed virtual community, for instance for elderly, members can discuss issues or chat with each other, exchange advises, and get to know new people in an environment that is safe and built for them. Furthermore, those people who are unable to attend gatherings and have face-to-face interactions, virtual communities provide benefits and opportunities to socialize. However, the maintenance of systems supporting this kind of interactions consumes effort, and unless some basic mechanisms exists that assist the administration tasks, these communities can not grown and evolve properly.

The overall goal of the TeleCARE project is the design and development of a configurable framework solution, for tele-supervision and tele-assistance, to support the elderly (TeleCARE Consortium, 2003). It benefits from and is based on the integration of: (i) multi-agent systems (MAS), including both stationary and mobile intelligent agents, (ii) federated information management, and (iii) the services that 
are likely to be offered by the emerging ubiquitous computing and intelligent home appliances. The Core horizontal platform developed for TeleCARE provides the MAS and the federated information management services. The TeleCARE project further develops some vertical services on top of this platform, including status monitoring, as well as other forms of assistance such as agenda reminders, entertainment services, time bank, and a base service for virtual community.

This paper describes the design of agent-based VC Support service on top of the TeleCARE infrastructure. The VC Support service will be the base for many other vertical services developed in TeleCARE, including the time bank and value-added entertainment services.

The paper is organized as follows, first the virtual community paradigm is described, and its roles and benefits are addressed. Then, major requirements and design issues related to a VC Support service for elderly in TeleCARE are described. Finally, conclusions and future work are drawn at the end of the paper.

\section{VIRTUAL COMMUNITIES}

The following definition of Virtual Community (VC) by Rheingold in (Rheingold, 1993) is widely accepted and quoted in many other publications and discussions:

"Virtual communities are social aggregations that emerge from the Net when enough people carry on public discussions long enough, with sufficient human feeling, to form webs of personal relationships in cyberspace".

This definition further describes the Net as "the loosely interconnected computer networks that link people around the world together", and refers to cyberspace as representing "the conceptual space where people manifest data, information, words, human relationships, wealth, and power, using the Net". This spatial metaphor creates a sense of place or locale in which people feel present or co-present with others (Turkle, 1995).

Today, the term "online community" is also being used referring the VC, and for our purposes this term is a synonym to the definition above (Kim, 2000;McArthur and Bruza, 2001). Thus basically, an online or virtual community is a gathering of people in a cyberspace where they join, communicate, connect, and get to know each other better over time.

Virtual communities are in fact born immediately at the beginning of the Internet availability. However, at the beginning, these were mostly online communities with a simple agreement to use the electronic bulletin boards (BBS), or some unsophisticated operating system tools, such as 'talk' command in Unix, that supported connecting people across geographical regions. Ever since, the evolution of virtual communities, many systems and tools have been developed, most of them even free for public use such as: chat rooms, forums, discussion boards, newsletters, etc., that give people more possibilities to socialize.

Furthermore, gradually online communities have become focused in a wide range of classified interests and motivations. This extends the communities toward specific topics or purposes for which many different interest groups can be built. Nowadays, virtual communities are extremely popular; and it is nearly impossible to track the number of these online communities around the world such as (SeniorNet, 2003; SeniorWeb, 2003; ThirdAge Inc., 2003). 


\subsection{Online activities within a Virtual Community}

There are several activities that can be carried out within a VC, once it has been established. The following Table 1 shows some of the main types of activities people commonly use through VCs (Boetcher, et al., 2002).

Table 1 - Main types of VC activities
\begin{tabular}{|c|l|}
\hline Activity & Description \\
\hline $\begin{array}{l}\text { Joint interest } \\
\text { groups }\end{array}$ & $\begin{array}{l}\text { Virtual communities play an important role for joining people who share common interests in issues } \\
\text { and causes. Support groups for people dealing with certain diseases, as well as interest in politics, laws, } \\
\text { religion, city regulations or the environment, or people interested in studying together, can each form a } \\
\text { nucleus for a VC. }\end{array}$ \\
\hline $\begin{array}{l}\text { Joint work } \\
\text { (business). }\end{array}$ & $\begin{array}{l}\text { Distributed work groups within companies and between companies use online community of people to } \\
\text { build their team, keep in touch and even work on projects together. A very detailed description of how } \\
\text { online work groups are established to work through the VC can be found at http://www.co-i- } \\
\text { l.com/coi//knowledge-garden/cop/index.shtml and http://www.awaken.com }\end{array}$ \\
\hline Socialize & $\begin{array}{l}\text { Meeting people online, playing, sharing jokes, story telling and just taking interest in each other. } \\
\text { Communities tike this often focus around bulletin boards and chat rooms. An example of such a } \\
\text { community is MSN Site at http://www.msn.com }\end{array}$ \\
\hline Joint activities & $\begin{array}{l}\text { It offers to communities ways to communicate and join together in activities. Community groups such } \\
\text { as soccer teams and school groups and others have used online community to provide forums for } \\
\text { information and discussion, helping to bring groups together. }\end{array}$ \\
\hline $\begin{array}{l}\text { Topical } \\
\text { conversation } \\
\text { groups }\end{array}$ & $\begin{array}{l}\text { Online salons and discussion forums such as the Well (http://www.well.com), Salon's TableTalk } \\
\text { (http://www.salon.com), Cafe Utne (http://www.utne.com) and others have formed communities of } \\
\text { people who enjoy conversations about certain topics and shared interests }\end{array}$ \\
\hline
\end{tabular}

\subsection{Building Virtual Communities}

When building a virtual community, one of the most important decisions is the selection of the community platform and its management approach. Restrictions such as time, budget, openness or autonomy may determine these decisions.

Some potential solutions for the community platform include: (i) using the services of community service providers, (ii) leasing or purchasing community software to gain more control over the community platform, or (iii) develop proprietary community tools and infrastructure.

No matter which approach is selected, any virtual community requires suitable administration support in order to provide a good foundation for its building and maintenance. Primarily, the administration tasks can be divided into two major categories of operational administration and content/services management (Boetcher, et al., 2002).

The operational administration involves the provision of necessary hardware and software capabilities and other administration duties for the sake of the virtual community such as the server preparation and maintenance, hosting software tools, $\log$ facility provision, user member administration, etc.

Depending on the purpose of the VC, its potential size and other characteristics of the community; and the chosen support to provide, content/services management needs to be supported. For instance, mechanisms are needed to support the possibility that may have a wide rage, including: add new discussion topic, start new interest group, provision of chat channels, and the ability to store, edit, hide or delete messages, track participation information, develop and provide newsletter, etc. 


\subsection{Tools for Virtual Communities}

There are already a number of free software tools (and services) that can be used in order to set up a virtual community. A virtual community can be organized and administered with many alternative mechanisms and can support a variety of functions that vary in what they provide and in their format. Many existing community services support some form of chat, email, web pages, etc. However, the selection of the right tools to provide is related to the audience as well as the main purpose of the VC.

Table 2 below presents a list of major existing community-oriented support mechanisms and tools, which are provided through online communities. However, note that this list is selective, rather than exhaustive due to the highly dynamic nature of this field.

Table 2 - VC support mechanisms

\begin{tabular}{|l|l|}
\hline Name & Description \\
\hline Email & $\begin{array}{l}\text { This tool can send and receive messages directly to the inbox of the recipient(s). The email message can } \\
\text { be also individualized. Email newsletters are used for one-to-many communication, which allow the } \\
\text { information provision and update for a large group of users quickly and cost effectively. }\end{array}$ \\
\hline $\begin{array}{l}\text { Mailing list } \\
\text { establishment }\end{array}$ & $\begin{array}{l}\text { Mailing lists support emails sent to a group of email addresses at once. The main applications are for one- } \\
\text { to many or for many-to-many communications like newsletters where each member of a list can also } \\
\text { respond to the entire group. }\end{array}$ \\
\hline $\begin{array}{l}\text { Discussion } \\
\text { boards }\end{array}$ & $\begin{array}{l}\text { This mechanism supports web-based asynchronous conversation spaces for their members. These spaces } \\
\text { are also known as bulletin boards, online forums or conferences. }\end{array}$ \\
\hline Chat & $\begin{array}{l}\text { A chat tool offers synchronous online interaction among the users. It can be a useful tool to provide } \\
\text { effective small group meetings, where decisions can be made and details arranged }\end{array}$ \\
\hline $\begin{array}{l}\text { Instant } \\
\text { messaging }\end{array}$ & $\begin{array}{l}\text { Instant messaging tools allow their members to see who is online at any moment, and send spontaneous } \\
\text { and unplanned messages to them }\end{array}$ \\
\hline $\begin{array}{l}\text { Internet } \\
\text { broadcasts }\end{array}$ & $\begin{array}{l}\text { The possibility to stream media (audio and video) using multimedia technologies, allow a one-to-many } \\
\text { presentation }\end{array}$ \\
\hline $\begin{array}{l}\text { Some } \\
\text { traditional tools }\end{array}$ & $\begin{array}{l}\text { Further range of tools, including traditional methods such as tele-conferencing, video-conferencing, and } \\
\text { other similar media can be considered in combination with online mechanisms }\end{array}$ \\
\hline
\end{tabular}

\section{THE TELECARE PLATFORM}

This section focuses on the multi-agent TeleCARE platform and its design principles, and how the planned infrastructure is linked with the development of a base service to support virtual communities.

\subsection{The TeleCARE project}

The IST 5FP TeleCARE project aims at design and development of a configurable framework focused on the elderly support. The proposed solution has to be seen as complementary to other initiatives, focused on the integration of elderly in the society to reduce their isolation. The TeleCARE consortium involves research organizations, technology developers, socio-economic experts, and elderly support organizations from Portugal, Spain, United Kingdom, and the Netherlands (TeleCARE Consortium, 2003).

Due to the constant changes in global market, new value-added vertical services are needed to be gradually developed and applied to the TeleCARE environment, while easing the TeleCARE administration. The TeleCARE solution benefits from the merge of a number of technologies and paradigms in order to provide an open architecture supporting seamless future expansion of the TeleCARE services. The 
main areas of research approaches and advanced technology included consists of: Multi-agent systems and mobile agents, Federated database management systems, interoperability support and data exchange defined with proper ontologies, advanced user interfaces, safe communications and authentication mechanisms, among others.

\subsection{Enabling approaches and technologies}

This subsection provides a brief description of the main approaches and the two technologies applied to TeleCARE design and development that are most closely related to the VC Support service (Section 4).

\section{Multi-agent systems}

During the last decade, the Multi-Agent System (MAS) technology has advanced to the level that it can offer many potential advantages including robustness, efficiency, flexibility, adaptability, scalability, inter-operation of multiple existing systems, reliability and extensibility.

The TeleCARE infrastructure is based on the Aglets, a multi-agent system based on Java (Aglets.org, 2002;IBM Tokyo Research Laboratory, 2002). The TeleCARE platform supports seamless interactions among stationary and mobile intelligent agents, with planning, error diagnosis, and recovery capabilities. Agents in TeleCARE are able to recognize and self-adapt to diverse environments. At the same time, the mobile agent technology provides a rich base for information brokerage in networked environments (Wang, et al., 1999) and a possible approach for implementing the federated information management systems (Papastavrou, et al., 1999), as it is applied in TeleCARE and described below.

\section{Federated information management}

The mechanism addressing information interoperation among sites in TeleCARE must consider the sharing and exchange of distributed information in a highly heterogeneous environment (Afsarmanesh and Camarinha-Matos, 1997;Busse, et al., 1999;Camarinha-Matos, et al., 2001; Sheth and Larson, 1990). The federated approach to information management in networks of independent cooperative nodes is well suited to management of all local and distributed information handled in TeleCARE, while preserving the node autonomy and information privacy. The federated query processing mechanisms allow access to distributed information according to their defined access rights, where TeleCARE sites represent autonomous nodes in the federation. Namely, TeleCARE nodes run independent of each other, and may evolve independently in terms of applications, devices, vertical services and data models.

The TeleCARE platform applies the results gained in previous research and development projects, in specific from ESPIRIT II - ARCHON (Afsarmanesh and Tuijnman, 1992) and ESPIRIT IV - PRODNET II (Garita, et al., 2000) for federation of autonomous and heterogeneous expert systems. Federated information management applied to Virtual Organizations supports the cooperation between the nodes in the federation to accomplish a common or global task, while the local autonomy and independence of every node is preserved and reinforced. 


\section{THE PROPOSED VC SUPPORT SERVICE}

The flexible architecture of the TeleCARE system, based on adaptive mobile agents and information federation mechanisms, is described in (Camarinha-Matos and Afsarmanesh, 2002). For TeleCARE, a base service supporting the Virtual Community (VC) is being developed. This VC Support service provides the base mechanism for administration and evolution of TeleCARE virtual communities. As such, this tool provides basic functionalities to support the creation, operation, evolution and maintenance of virtual communities in the TeleCARE environment.

This VC Support is mainly a base service that will be used for the design and development of other value-added TeleCARE vertical services (e.g. time bank, entertainment services, etc.). The VC support enables the maintenance of reliable services for the community members in the TeleCARE network, in an integrated manner for both the VC administrators and the vertical value-added service providers of TeleCARE; thus avoiding the need for establishment of isolated and repetitive VC related services.

The remaining of this section focuses on the design principles behind the VC Support base service, and how it is linked to the TeleCARE platform.

\subsection{VC Support overview}

The TeleCARE platform provides the base mechanisms and horizontal infrastructure for inter-linking of nodes in the TeleCARE network, e.g. elderly home, care centers, and other nodes in this environment. This enables both simple and advanced vertical services to be designed and developed with the focus on the service and the users, rather than on the basic infrastructure. Vertical services for TeleCARE mainly focus either on the assistance of end users with their activities as individuals, (e.g. elderly's agenda organizer or monitoring elderly at home, etc.) or as a member of a community (time bank, group activities planning, etc.).

In order to properly support the later group of vertical services providing community-related activities, there is a clear need for a base service providing the necessary functionality to support a VC throughout its life cycle. The VC Support in TeleCARE aims at the provision of such base functionality to support the creation, operation, administration and maintenance of a virtual community, on top of which other TeleCARE vertical services can be defined. Therefore, the VC for TeleCARE also extensively makes use of the agent approach and the federated information management provided though the base TeleCARE platform.

As presented in Figure 1, the VC Support is located between of the TeleCARE platform and the VC value added services, where it uses the platform functionalities, and provide support for the community value-added vertical services.

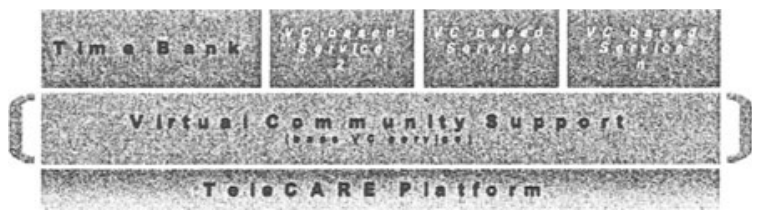

Figure 1 - VC Support service 
Therefore, the main objective of the VC Support base service is to enable vertical service developers and administrators with developing reliable VC-based vertical services, applications, and products on top of the TeleCARE platform in a uniform way. Moreover, the provision of VC Support as a base service reduces the complexity of vertical value-added services by taking advantage of integrated support functionalities.

\subsection{The VC model}

As depicted in Figure 2, in TeleCARE, the virtual communities created and administered by different organizations, e.g. a care center, a leisure site, etc., can support a number of VC-based services.

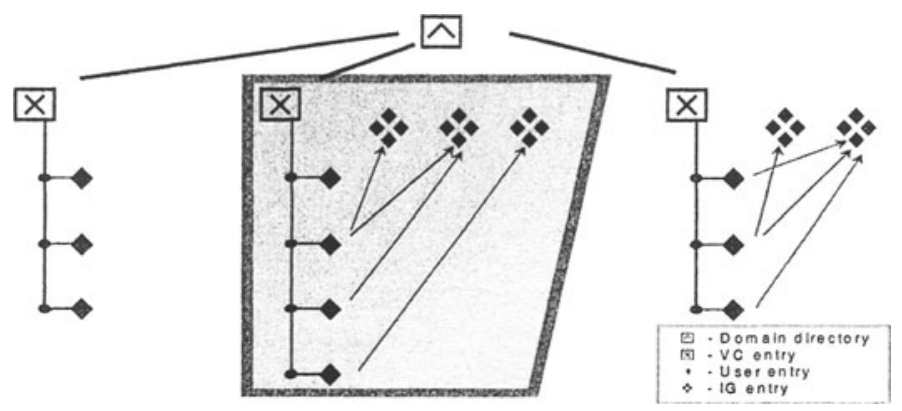

Figure 2 - Model of VC Support

Therefore, the virtual community service must support a set of basic configuration attributes, registered members and Interest Groups (IG). In every VC, the administrator can define several IGs and designate users, among the respective VC members, into these groups, as shown by the screen shots of this VC support system in Figure 3(a). Furthermore, the VC administrator can assign moderators to the IGs, which in turn can define one or more message boards for interaction and discussion, or for general announcements, for the benefit of the members of the respective IG, as shown in Figure 3(b).
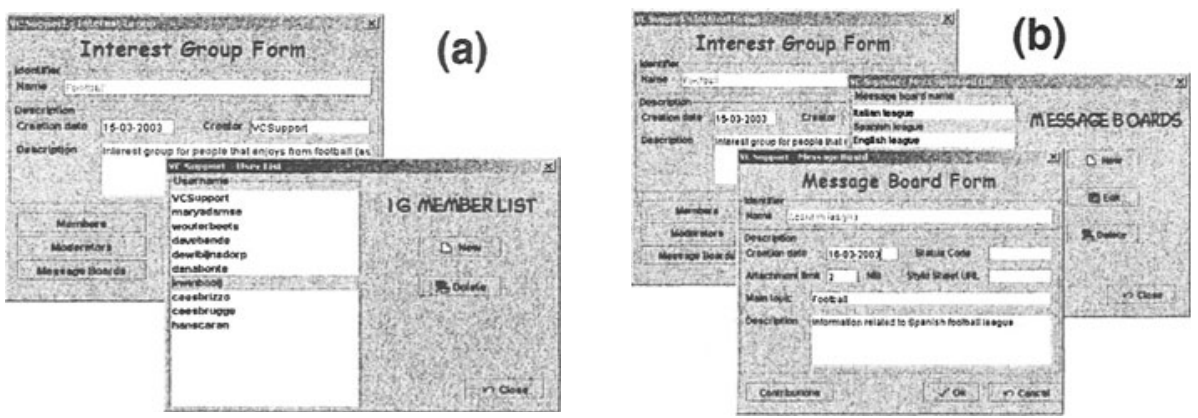

Figure 3 - VC Support administration 
Interactions within any $\mathrm{VC}$ are performed through its members' contributions (see Figure 4). Contributions contain articles or documents submitted by the VC members, and it is considered that only the moderator and the author of the contribution are allowed to modify the article.

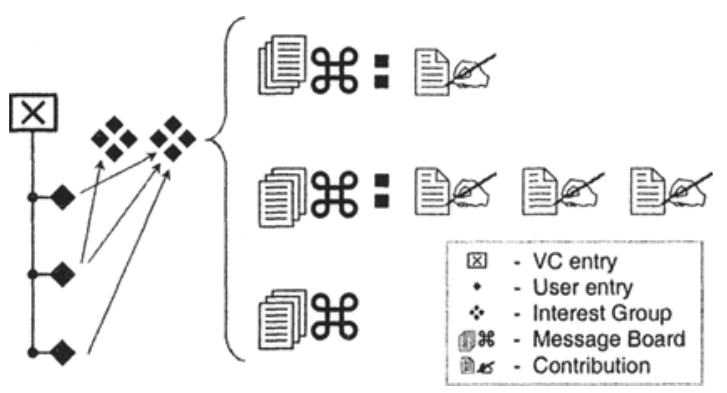

Figure 4 - VC message boards and their contributions

Also notice that the VC Support offers mechanisms to handle the submitted material. However, the task of creation/submission of content material for the message board is the responsibility of the VC-based service provider.

\subsection{VC Support service functionality}

As stated previously, the VC Support base service is located between the TeleCARE platform and the VC vertical services. This the functionality of the VC Support base service includes proper creation/operation of virtual communities, their members, and their contributions, and it is initially classified in two groups: (i) VC Configuration \& Administration and (ii) VC Services Management (see Figure 5).

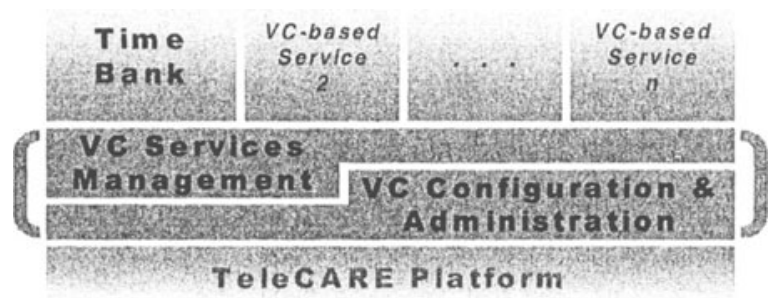

Figure 5 - VC Support functionality groups

The VC Configuration \& Administration supports the creation/dissolution of virtual communities, as well as their membership administration. Operations to establish particular VC settings, such as contact person/administrator definition, and activities that support evolution of the VC members, as listed in Table 3, are developed. Flexibility is a major design goal in this component since the VC services must support a range of communities, each with different purposes. 
Table 3 - VC Configuration \& Administration operations

\begin{tabular}{|c|c|}
\hline VC creation and dissolution administration & VC membership administration \\
\hline Create VC entry & Create user entry \\
\hline Update VC entry & Update user entry \\
\hline Remove VC entry & Remove user entry \\
\hline List VC entries & Bind user with VC \\
& Unbind user from VC \\
& VC member list \\
& Verify user VC membership \\
\hline
\end{tabular}

The VC Services Management supports the management of activities provided within the VC. These facilities provide operations for the definition and management of Interest Groups (IG) and Message Boards (MB) as presented in Table 4. The IG management is an integral part of the virtual communities and allows the grouping of the VC members in different context-based categories, according to their objectives or other classifications. Moreover, this area provides mechanisms for definition and updating of the information content within the message boards.

Table 4 - VC Services Management operations

\begin{tabular}{|l|c|c|}
\hline $\begin{array}{l}\text { Interest Groups } \\
\text { management }\end{array}$ & $\begin{array}{l}\text { Message Board } \\
\text { management }\end{array}$ & Content management \\
\hline Create IG entry & Create MB entry & Create contribution entry \\
\hline Update IG entry & Update MB entry & Update contribution entry \\
\hline Remove IG entry & RB list & Remove MB entry \\
\hline List IG entries & & Contribution list \\
\hline Bind user with IG & & Set contribution status \\
\hline Unbind user from IG & Attachment operations) \\
\hline IG member list & & \\
\hline Verify IG user membership & & \\
\hline
\end{tabular}

\section{CONCLUSIONS AND FUTURE WORK}

The TeleCARE platform, based on a multi-agent and mobile-agent architecture, is intended to facilitate the (distributed) administration of the VC processes. In addition, its federated information management supports the VC data modeling, storage, and retrieval.

In TeleCARE the VC Support will provide its end users (elderly) new approaches to socialize and improve their quality of life. The participation in virtual communities can be an important feature, playing a significant role in reviving personal experiences (Glogoff, 2001) that may be impossible otherwise. In TeleCARE, the VC Support offers the elderly, the feeling of belonging, communication, and possibility of contribution and collaboration within and through a community of elderly. The developed VC system supports the main functionality required for virtual communities in TeleCARE.

The VC Support is designed and developed as a base service within the TeleCARE environment. As such, this VC service benefits from the flexibility provided by both the MAS and federated information management paradigms for its implementation, and fit suitably with the TeleCARE Platform. At the same time, it facilitates the development of new vertical value-added services that also require the existence of a virtual community for their functionality. 
The next step for this work focuses on the definition of methodologies for (1) proper day-to-day administration of the VC, and (2) proper use of the VC base service for the development of other value-added vertical services.

\section{Acknowledgments}

This work was funded in part by the IST program of the European Commission. The authors thank the contribution of the TeleCARE consortium.

\section{REFERENCES}

1. Afsarmanesh, H. and Camarinha-Matos, L.M., Federated Information Management for Cooperative Virtual Organizations. in 8th International Conference on Databases and Expert Systems Applicactions, DEXA'97, (Toulouse, France, 1997), 561-572.

2. Afsarmanesh, H. and Tuijnman, F. The Information Modeling within the ARCHON Architecture. in ARCHON - An Architecture for Multi-Agent Systems., Ellis Horwood Publishers, 1992, 59-76.

3. Aglets.org. The Aglets Portal, http://aglets.sourceforge.net/, 2002.

4. Boetcher, S., Duggan, H. and White, N. What is a Virtual Community and Why Would You Ever Need One?, http://www.fullcirc.com/community/communitywhatwhy.htm, 2002.

5. Busse, S., Kutsche, R.-D., Leser, U. and Weber, H. Federated Information Systems: Concepts, Terminology and Architectures, Computergestutzte InformationssystemeCIS, Technische Universitat Berlin, Berlin, 1999.

6. Camarinha-Matos, L. and Afsarmanesh, H., Design of a Virtual Community for Elderly Support. in Infrastructures for Virtual Enterprises (PRO-VE'02), (Sesimbra, Portugal, 2002), Kluwer Academic Publishers.

7. Camarinha-Matos, L., Afsarmanesh, H., Kaletas, E. and Cardoso, T., Service Federation in Virtual Organizations. in Eleventh International IFIP TC5 WG-5.2 - WG-5.3 Conference, (Budapest, Hungary, 2001), Kluwer.

8. Garita, C., Afsarmanesh, H. and Hertzberger, L.O. The PRODNET Federated Information Management Approach for Virtual Enterprise Support. Journal of Intelligent Manufacturing.

9. Glogoff, S. Virtual Connections: Community Bonding on the Net. First Monday, 6 (3).

10. IBM Tokyo Research Laboratory. Aglets Workbench, http://www.trl.ibm.com/aglets/, IBM Japan, 2002.

11. Kim, A.J. Community Building on the Web. Peachpit Press, Berkeley, California, 2000.

12. McArthur, R. and Bruza, P. The ABC's of Online Community. Lecture Notes in Computer Science, 2198. 141--??

13. Papastavrou, S., Samaras, G. and Pitoura, E., Mobile Agents for WWW Distributed Database Access. in International Conference on Data Engineering (ICDE'99), (Sydney, Australia, 1999), 228 237.

14. Rheingold, $\mathrm{H}$. The virtual community: Homesteading on the electronic frontier. Addison-Wesley, 1993.

15. SeniorNet. SeniorNet - Bringing wisdom to the Information Age, http://www.seniornet.org, 2003.

16. SeniorWeb. SeniorWeb, http://www.seniorweb.nl, 2003.

17. Sheth, A. and Larson, J. Federated Database Systems for Managing Distributed, Heterogeneous, and Autonomous Databases. ACM Computing Surveys, 22. 183-236.

18. TeleCARE Consortium. A Multi-Agent Tele-Supervision System for Elderly Care, http://www.uninova.pt/ telecare, 2003.

19. ThirdAge Inc. ThirdAge, http://www.thirdage.com, 2003.

20. Turkle, S. Life On the Screen: Identity in the Age of the Internet. Simon and Schuster, New York, 1995.

21. Wang, A.I., Liu, C. and Conradi, R., A Multi-Agent Architecture for Cooperative Software Engineering. in International Conference on Software Engineering and Knowledge Engineering (SEKE'99), (Kaiserslautern, Germany, 1999), 1 - 22. 